\title{
Pilomatrixomas en niños: a propósito de 149 casos. Estudio retrospectivo en dos centros pediátricos
}

\author{
Pilomatrixomas in children: Report of 149 cases. A retrospective study at two \\ children's hospitals
}

\author{
Dr. Jorge A. Laffargue ${ }^{a}$, Dra. Paola C. Stefano ${ }^{b}$, Dra. Jésica L. Vivoda ${ }^{a}$ Dra. Mónica L. Yarza ${ }^{a}$, \\ Dra. Ana G. Bellelli, Dra. María N. Castro ${ }^{a}$, Dra. Adriana N. Torres Huamani ${ }^{b}$ Dr. Sebastián N. Apac, \\ Dr. Daniel Navacchia ${ }^{d}$ Dra. María Centeno ${ }^{e}$ Dra. Andrea B. Cervinib y Dra. Susana A. Grees ${ }^{a}$
}

\begin{abstract}
RESUMEN
El pilomatrixoma es un tumor benigno cutáneo, de frecuente aparición en los niños, que se origina en las células de la matriz del folículo piloso.

Se presenta como un nódulo o tumor, aproximadamente, de 0,5 a $3 \mathrm{~cm}$ de tamaño, de consistencia cálcica, de bordes facetados, con piel suprayacente eritematoazulada. En ocasiones, la piel puede atrofiarse, dar la apariencia de una ampolla e, incluso, extruir material cálcico.

El diagnóstico se sospecha por la presentación clínica característica y los estudios de imágenes complementarios. El diagnóstico definitivo es por los hallazgos histológicos. La extirpación quirúrgica es el tratamiento de elección.

Se presentan los datos epidemiológicos, clínicos, estudios complementarios y técnicas quirúrgicas empleadas de 149 pilomatrixomas resecados en 137 pacientes tratados en los Servicios de Dermatología de los hospitales de pediatría Dr. Pedro de Elizalde y Prof. Dr. Juan P. Garrahan.

Palabras clave: pilomatrixoma, neoplasias de anexos y apéndices cutáneos, niño, procedimientos quirúrgicos operativos.
\end{abstract}

http: / / dx.doi.org/10.5546/ aap.2019.340

Texto completo en inglés:

http:/ / dx.doi.org/10.5546/ aap.2019.eng.340

Cómo citar: Laffargue JA, Stefano PC, Vivoda JL, Yarza ML, et al. Pilomatrixomas en niños: a propósito de 149 casos. Estudio retrospectivo en dos centros pediátricos. Arch Argent Pediatr 2019;117(5):340-343.

a. Servicio de Dermatología Pediátrica del Hospital de Niños Pedro de Elizalde.

b. Servicio de Dermatología del Hospital de Pediatría "Prof. Dr. Juan P. Garrahan".

c. Servicio de Cirugía Plástica del Hospital de Pediatría "Prof. Dr. Juan P. Garrahan".

d. Servicio de Anatomía Patológica del Hospital de Niños Pedro de Elizalde.

e. Servicio de Anatomía Patológica del Hospital Nacional de Pediatría "Prof. Dr. J. P. Garrahan".

Ciudad Autónoma de Buenos Aires, Argentina.

Correspondencia:

Dr. Jorge Laffargue: drjorgealaffargue@gmail.com

Financiamiento: Ninguno.

Conflicto de intereses: Ninguno que declarar.

Recibido: 16-9-2018

Aceptado: 10-4-2019

\section{INTRODUCCIÓN}

El pilomatrixoma es una neoplasia benigna que se origina en las células de la matriz de los folículos pilosos. Se desconoce con exactitud su origen $y$, si bien se ha asociado a traumatismos y picaduras de insectos, en los últimos años, se ha encontrado una mutación activadora de la $\beta$-catenina en el $75 \%$ de los pilomatrixomas, que podría ser la causante de esta entidad. Es frecuente en los niños, en las primeras dos décadas de la vida. ${ }^{1}$

El pilomatrixoma se presenta, clásicamente, como un nódulo o tumor solitario, de consistencia dura, de bordes facetados, con piel suprayacente normocoloreada o eritematoazulada. Suele localizarse en la cara y el cuello, aunque también se encuentra en los miembros. Crece con lentitud y se estabiliza luego de meses o años. Por lo general, permanece asintomático, pero puede presentar inflamación y dolor. ${ }^{2,3}$

Existen otras formas clínicas de presentación menos frecuentes. En la variante anetodérmica o seudoampollar, el pilomatrixoma se encuentra cubierto por piel atrófica, eritematosa y con telangiectasias. ${ }^{4}$ En raras ocasiones, crece rápidamente, produce una úlcera y extruye material cálcico, lo que se denomina pilomatrixoma perforante. ${ }^{5}$ Otra variedad infrecuente es el pilomatrixoma gigante, definido como aquel que mide más de $5 \mathrm{~cm} .{ }^{6}$

En ocasiones, el paciente puede presentar múltiples pilomatrixomas. Existen casos familiares de pilomatrixomas múltiples y múltiples asociados a síndromes, como el síndrome de Turner, el síndrome de desajuste de reparación constitucional (Constitutional Mismatch Repair Deficiency; $C M M R-D$, por sus siglas en inglés), el síndrome de Gardner, la distrofia miotónica y el síndrome de Kabuki. ${ }^{78}$

El estudio ecográfico de la lesión permite acercar el diagnóstico en los casos en los que las manifestaciones clínicas generan dudas. Puede evidenciarse una imagen bien delimitada, con halo hipoecogénico periférico, con 
microcalcificaciones aisladas o parcialmente calcificado, o bien una imagen nodular calcificada en su totalidad con intensa sombra acústica posterior. ${ }^{9}$

La histopatología confirma el diagnóstico, en la cual se observan tres tipos de células. Las células basófilas o basaloides periféricas, que son células inmaduras de la matriz del folículo piloso con núcleo grande central y numerosas mitosis; las transicionales con citoplasma acidófilo y las células sombra o fantasma sin núcleo. Otros hallazgos que pueden encontrarse son la presencia de una reacción granulomatosa con células gigantes multinucleadas, depósitos de calcio y / u osificación. ${ }^{2}$

El único tratamiento posible es la extirpación quirúrgica. En los pilomatrixomas muy pequeños, asintomáticos, que no causen alteración estética o en niños de corta edad, la cirugía puede diferirse. ${ }^{2}$

Existen diferentes técnicas quirúrgicas, y se puede realizar la exéresis por losange o por avulsión. Esta última consiste en realizar una incisión en el centro del pilomatrixoma y tomarlo entre el dedo índice y el pulgar para provocar su extrusión. Se realiza el socavado con pinza metzenbaum y se sigue ejerciendo presión. Esta técnica disminuye el tiempo de la cirugía en comparación con la técnica por losange y tiene menor tasa de recidiva y mejores resultados estéticos. ${ }^{10}$ Las complicaciones posteriores a la cirugía pueden ser la sobreinfección de la herida, la aparición de un hematoma, la dehiscencia de la sutura, la cicatriz hipertrófica o queloidea.

El objetivo de este estudio es presentar los datos epidemiológicos, clínicos, estudios complementarios y tratamiento de los pilomatrixomas resecados en dos centros hospitalarios pediátricos.

Tabla 1. Datos epidemiológicos y clínicos

\begin{tabular}{ll}
\hline Variable & Número total \\
\hline Sexo & Femenino: 74 \\
& Masculino: 63 \\
Edad & De 9 meses a 18 años (media de 9,5 años) \\
Clínica & 133 clásicos \\
& 11 anetodérmicos \\
& 3 perforantes \\
& 2 gigantes \\
Localización & El 39,6\%, en los miembros superiores \\
& El $37 \%$, en la cabeza y el cuello \\
& El $20 \%$, en el tronco \\
& El 3,4 $\%$, en los miembros inferiores
\end{tabular}

\section{DISEÑO}

Estudio observacional, retrospectivo y descriptivo.

\section{MATERIAL Y MÉTODOS}

Se realizó la revisión de las historias clínicas de los pacientes con diagnóstico de pilomatrixoma atendidos en los Servicios de Dermatología de los hospitales de pediatría Dr. Pedro de Elizalde y Prof. Dr. Juan P. Garrahan entre marzo de 2011 y marzo de 2016. Se incluyeron todos los pacientes con edad entre 0 meses y 18 años, operados en alguno de los centros pediátricos mencionados, con confirmación diagnóstica histopatológica de pilomatrixoma. Se excluyeron aquellos pacientes con pilomatrixomas que no continuaron en seguimiento.

Se analizaron las siguientes variables: edad de aparición, sexo, presentación clínica del pilomatrixoma (clásica, papular, nodular, tumoral, anetodérmico, perforante), localización, tamaño, número de lesiones en cada paciente, número de recurrencia de lesiones, presencia de antecedentes familiares de pilomatrixoma, así como síndromes asociados. Se evaluaron los exámenes complementarios solicitados, los hallazgos histológicos y el tratamiento instaurado. El estudio fue aprobado por los Comités de Ética de ambos hospitales.

\section{RESULTADOS}

Se registró un total de 149 pilomatrixomas resecados a 137 pacientes (Tabla 1 ). De ellos, 74 eran de sexo femenino (el $54 \%$ ). El rango de edad fue de 9 meses a 18 años, con una media de 9,5 años.

La mayoría de los pacientes tuvieron un solo pilomatrixoma, pero 9 (el 6,57\%) presentaron más de uno. En ninguno de ellos, se hallaron antecedentes familiares de pilomatrixoma ni enfermedades asociadas.

Desde el punto de vista clínico, la mayoría de ellos (el $89 \%$ ) se presentó como la forma clásica en sus variantes nodular, papular y tumoral. Del resto, 11 fueron anetodérmicos; 3, perforantes; 2, gigantes (Figuras 1, 2 y 3).

La mayoría se localizó en los miembros superiores (el $39,6 \%$ ) y en el cuello y la cara (el $37 \%$ ), luego en el tronco (el $20 \%$ ) y solo el $3,4 \%$ en los miembros inferiores.

En 133 tumores, se solicitó ecografía, y, en 124 de ellos (el $93 \%$ ), se observaron imágenes con microcalcificaciones o imágenes totalmente calcificadas con sombra acústica posterior, 
compatibles con pilomatrixoma. Todos los pilomatrixomas resecados fueron evaluados por los Servicios de Anatomía Patológica, con hallazgos característicos.

Se realizó la exéresis por avulsión en la mayoría de los casos y la exéresis por losange en los anetodérmicos y perforantes (el $9 \%$ ). En ningún caso, se presentaron complicaciones posquirúrgicas. Se pudieron constatar recurrencias en un solo paciente (el 0,6\%).

\section{DISCUSIÓN}

Este es el primer estudio retrospectivo descriptivo de casuística de pilomatrixomas realizado en dos hospitales pediátricos en Latinoamérica. Cabe destacar que nuestra casuística es la más numerosa publicada hasta el momento. ${ }^{11-15}$

Si bien el pilomatrixoma tiene un pico de incidencia en las dos primeras décadas de la vida y otro pico a los 50-60 años, en nuestro estudio, la edad más temprana de aparición fue de 9 meses y la media fue de 9,5 años. En cuanto a la localización más frecuente reportada en la bibliografía, es en la cabeza y el cuello;-8-11 en nuestro estudio, fue más frecuente en los miembros superiores. La predominancia en el sexo femenino coincidió con la reportada en la bibliografía. $^{2}$

En nuestro estudio, la forma clásica de pilomatrixoma fue la más frecuente, lo que coincidió con lo reportado. ${ }^{2}$ La segunda variedad más frecuente en nuestro estudio fue

Figura 1. Pilomatrixoma de variante clásica

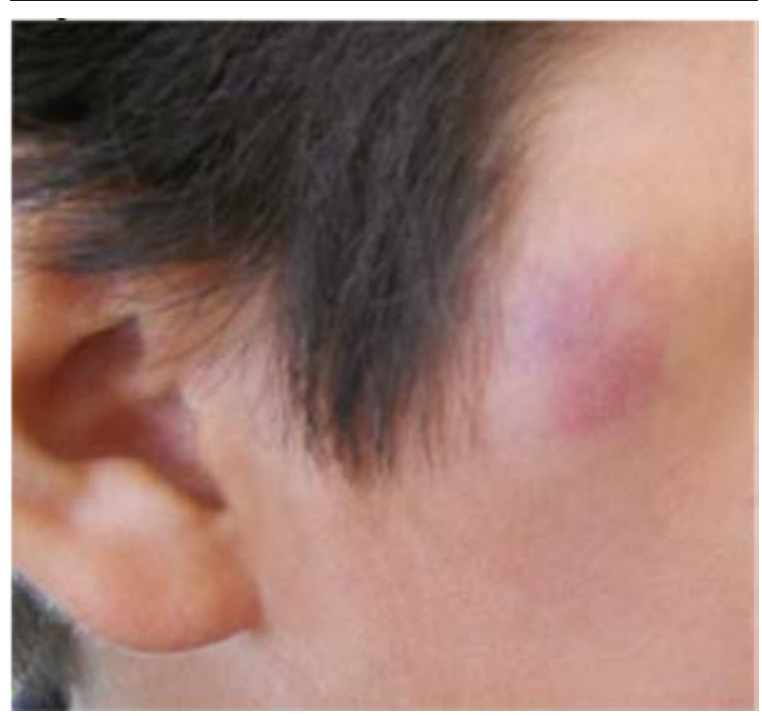

la anetodérmica. No se encontraron antecedentes de traumatismos en esta variedad. Los tres casos de pilomatrixomas perforantes ya se presentaron así en el momento de consulta y tampoco tenían antecedentes de traumatismo previo. En nuestro estudio, solo 2 pacientes presentaron la variedad clínica de pilomatrixoma gigante. Ninguno tuvo infección agregada.

Si bien la tasa de recidiva reportada es del 0-6 \%, , $^{11}$ en nuestro estudio, solo recidivó un pilomatrixoma hasta el seguimiento actual.

En una última revisión de la literatura sobre pilomatrixomas en niños y la asociación con otras anomalías, el $9 \%$ de los pacientes tenían pilomatrixomas múltiples, y, de estos, el $17 \%$ tenía anomalías asociadas. ${ }^{8}$ La asociación más

FIGURA 2. Pilomatrixoma anetodérmico o seudoampollar

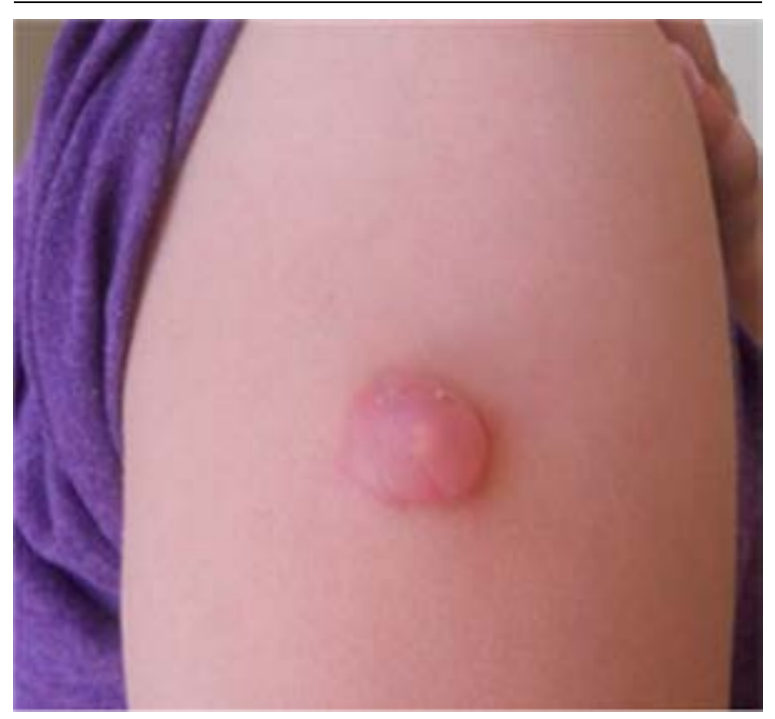

FIgURA 3. Pilomatrixoma perforante

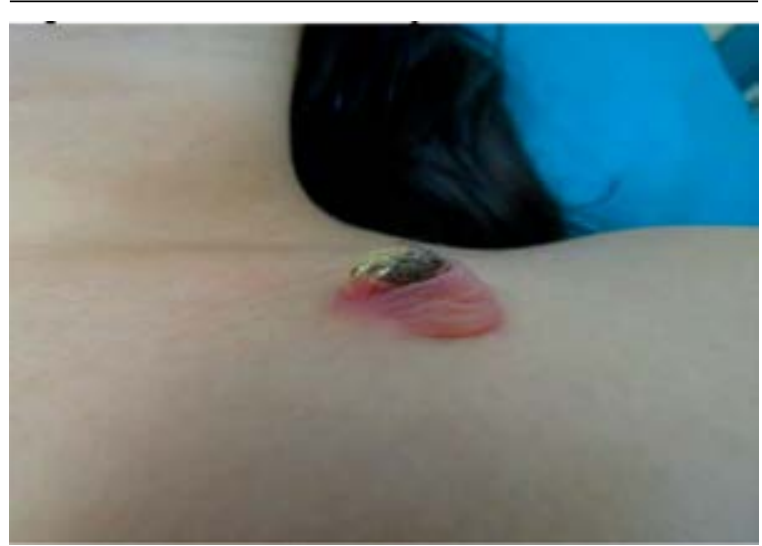


frecuente fue el síndrome de Turner, seguida por el $C M M R-D$, luego por el síndrome de Gardner y la distrofia muscular de Steinert. También se han reportado pilomatrixomas solitarios en el síndrome de Kabuki.

Además, existen pacientes con pilomatrixomas múltiples familiares no asociados a otras enfermedades. Los autores enfatizan que, si bien estas asociaciones son infrecuentes y que la mayoría fueron diagnosticadas antes de la aparición de los pilomatrixomas, se recomienda buscarlas en todo paciente con pilomatrixoma solitario o múltiple y realizar un seguimiento en el tiempo.

En nuestro estudio, el 6,7\% de los pacientes tuvo más de un pilomatrixoma en su evolución y ninguno presentó antecedentes familiares ni enfermedades asociadas durante el seguimiento hasta la fecha. No existe un tiempo consensuado y sugerido de seguimiento de los pacientes, pero sí es importante realizar una anamnesis completa sobre los antecedentes personales y familiares, y llevar a cabo un examen físico completo en búsqueda de signos presentes en los síndromes asociados a pilomatrixomas ya mencionados. ${ }^{8}$

Cabe destacar que, durante la exéresis del tumor en ambas técnicas quirúrgicas, se evidencia una hemorragia en napa y que, al extraer todo el material cálcico, cede. Este signo, que se ha visto en todas las exéresis realizadas en este trabajo, se ha denominado signo de la ausencia de sangrado. ${ }^{10}$ Se cree que podría deberse a que el pilomatrixoma es un tumor de localización superficial, que no invade el tejido celular subcutáneo.

Se considera importante conocer las distintas formas de presentación y evolución de los pilomatrixomas en niños, ya que es una tumoración de frecuente aparición en la infancia, así como también tener en cuenta las posibles asociaciones. Dependiendo de la forma de presentación, el tamaño y la velocidad de crecimiento, se decidirá el momento de realizar el tratamiento, que siempre es quirúrgico.

\section{REFERENCIAS}

1. Hassnein AM, GlanzSM. Beta-catenin expression in benign and malignant pilomatrix neoplasms. Br J Dermatol. 2004; 150(3):511-6.

2. Hoeger PH. Differencial diagnosis of skin nódulos and cysts. In: Irvine AD, Hoeger PH, Yan AC (eds.). Harper's Textbook of Pediatric Dermatology. 3rd ed. Oxford: Willey Blackwell; 2011.Págs.92.1-6.

3. Grinspan Bozza NO. Signo de la piedra facetada. Diagnóstico del pilomatrixoma. Arch Argent Dermatol. 2007; 57(4):203-5.

4. Concha MR, Farías MM, Abarzúa A, Droppelmann N, et al. Pilomatrixoma: una presentación clínica inusual. Arch Argent Pediatr. 2011; 109(6):e116-8.

5. Ces Costa M, Arias M, Bounsante M, Abeladaño A, et al. Lesión tumoral en muslo. Arch Argent Dermatol. 2007; 58(2):75-7.

6. Stefano P, Apa S, Balancini B. Pilomatrixoma gigante y ulcerado en un niño. Actas Dermasifiliogr. 2013; 104(10):92439.

7. García-Arpa M, Vera-Iglesias E, García Rojo M, RomeroAguilera G, et al. Pilomatrixomas múltiples. Med Cutan Iber Lat Am. 2010; 38(1):48-51.

8. Richet C, Maza A, Dreyfus I, Bourrat E, et al. Childhood pilomatricomas: associated anomalies. Pediatr Dermatol. 2018; 35(5):548-51.

9. Garioni E, Danesino G, Madonia L. Pilomatricoma: sonographic features. J Ultrasound. 2008; 11(2):76-8.

10. LaffargueJA, MeredizJ,StefanoP. Dermatología quirúrgica pediátrica. En De Carli E, Jaled M, Coringrato M, Galimberti $\mathrm{G}$, et al. Dermatología quirúrgica y estética. Buenos Aires: Journal; 2014.Págs.105-14.

11. Cigliano B, Baltogiannis N, De Marco M, Faviou E, et al. Pilomatricoma in childhood: a retrospective study from three European paediatric centres. Eur J Pediatr. 2005; 164(11):673-7.

12. Pulvermacker B, Seroussi D, Haddad R, Mitrofanoff M. Pilomatricome ou épithéliome calcifié de Malherbe: a propos d'une série de 89 cas chez l'énfant. Ann Chir Plast Esthet. 2007; 52(1):39-42.

13. Cypel TKS, Vijayasekara V, Somers GR, Zuker RM. Pilomatricoma: Experience of the hospital for sick children. Can J Plast Surg. 2007; 15(3):159-61.

14. Hassan F, Stephens E, Fallon S, Schady D, et al. Characterizing pilomatricomas in children: a single institution experience. J Pediatr Surg. 2013; 48(7):1551-6.

15. Kwon D, Grekov K, Krishnan M, Dyleski R. Characteristics of pilomatrixoma in children: a review of 137 patients. Int J Pediatr Otorhinolaryngol. 2014; 78(8):1337-41. 